\title{
Modeling Hysteresis of Ferrite Core Materials using Permeance-Capacitance Analogy for System-Level Circuit Simulations
}

\author{
M. Luo, D. Dujic, and J. Allmeling
}

This material is posted here with permission of the IEEE. Such permission of the IEEE does not in any way imply IEEE endorsement of any of EPFL's products or services. Internal or personal use of this material is permitted. However, permission to reprint / republish this material for advertising or promotional purposes or for creating new collective works for resale or redistribution must be obtained from the IEEE by writing to pubs-permissions@ieee. org. By choosing to view this document, you agree to all provisions of the copyright laws protecting it. 


\section{Modeling Frequency Independent Hysteresis Effects of Ferrite Core Materials using Permeance-Capacitance Analogy for System-Level Circuit Simulations}

Min Luo, Member, IEEE, Drazen Dujic, Senior Member, IEEE, and Jost Allmeling, Member, IEEE

\begin{abstract}
Ferrite materials are widely used for magnetic cores in power electronic converters. The hysteresis effect of the material leads to power loss and harmonic distortion. In order to predict the behaviour of the magnetic component in the system environment during the design phase, accurate system-level timedomain simulation is desired. This work proposes an approach to model the frequency-independent magnetic hysteresis effect of ferrite core materials in magnetic circuits based on the permeance-capacitance analogy. The model is able to accurately reproduce the per-cycle energy loss and equivalent permeability of the hysteresis loops under excitation in a wide range of amplitudes.
\end{abstract}

Index Terms-hysteresis, ferrite material, magnetic circuit, permeance-capacitance

\begin{tabular}{ll} 
& \multicolumn{1}{c}{ NOMENClAtURE } \\
$B$ & Flux density. \\
$B_{r}$ & Remanent flux density. \\
$H$ & Field strength. \\
$\Phi$ & Magnetic flux. \\
$\mathscr{P}$ & Magnetic permeance. \\
$\mu$ & Magnetic permeability.
\end{tabular}

\section{INTRODUCTION}

Ferrite materials are widely used to produce magnetic cores for inductive filters and isolation transformers in power electronic converters. In comparison to the ferromagnetic materials like amorphous alloy and nanocrystalline, ferrite materials have significantly lower conductivity so that the frequencyindependent magnetic hysteresis effect usually dominates the core loss. Due to the fact that the magnetic hysteresis contributes to the nonlinearity of the magnetic component's inductivity, which interacts with the remaining part of the power electronic system, the hysteresis loss is essentially a coupled effect. In order to predict the behaviour of the magnetic component during design phase, accurate time-domain hysteresis model which can be easily integrated into the system-level circuit environment and simulated in a fully coupled way is desired.

M. Luo and D. Dujic are with the Power Electronics Laboratory, École Polytechnique Fédérale de Lausanne (EPFL), Lausanne, CH-1015 Switzerland (e-mail:min.luo@epfl.ch,drazen.dujic@epfl.ch).

J. Allmeling is with Plexim GmbH, Zürich, CH-8005 Switzerland (e-mail: allmeling@plexim.com).

Manuscript received November 30, 2017; accepted Feburary 7, 2018.

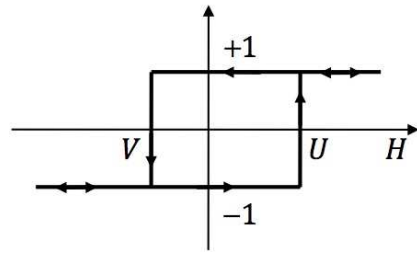

Fig. 1: Single hysteron $\gamma(U, V)$ of Preisach Model.

The permeance-capacitance analogy approach, which was proposed in the work of [1], provides a practical way to combine magnetic circuit seamlessly into system-level simulation. The benefit in modeling complex magnetic structures using permeance-capacitor approach has been demonstrated by the authors of [2], and the authors of [3] have introduced this approach into a commercial system-level simulation tool for power electronics. Further on, this approach has been applied in the work of [4], [5] to capture different magnetic phenomena. In this work, we also choose the permeancecapacitor approach as fundamental platform for the modeling.

For time-domain simulation of frequency-independent magnetic hysteresis, Preisach model has been recognised as a flexible approach especially regarding its ability to capture minor loops [6]. According to the formulation of the scalar Preisach model, the magnetic hysteresis is subdivided into many small independent particles [7], called hysterons. Each square-loop hysteron $\gamma(U, V)$ switches between -1 and +1 at a unique set of transition boundaries $U$ and $V$, depending on the applied field strength $H$ as well as its history, as demonstrated in Fig. 1. The flux density $B$ is expressed as the weighted summation of all hysterons using a probability distribution function (PDF) $p(U, V)$, or in other words, Everett integration. In the case of soft magnetic materials like ferrite, the twodimensional distribution function $p(U, V)$ can be expressed as the product of two one-dimentional PDFs, considering the fact that the probability of a hysteron switching in one direction is essentially independent of that switching in the opposite direction [8]. 


$$
\begin{aligned}
& B=\iint p(U, V) \cdot \gamma(U, V) \cdot d U d V \\
&=\iint p_{s}(U) \cdot p_{s}(-V) \cdot \gamma(U, V) \cdot d U d V
\end{aligned}
$$

Authors of [9] proposed Cauchy-Lorentz PDF for the distribution function $p_{s}$, which was further applied by the work of [10], [11] and [12] for modeling of soft magnetic materials. A parameter identification scheme using genetic algorithm has been presented in [13]. Taking advantage of the feature that the Cauchy-Lorentz PDF has closed-form integral, the differential permeability $\mu=d B / d H$ can be analytically calculated, so that the Preisach model was able to be directly applied to the permeance-capacitor magnetic circuit model in the work of [3]. In the existing publications, verification of the Preisach model with Cauchy-Lorentz PDF has only been carried out on metal based material like silicon steel. For ferrite however, Cauchy-Lorentz PDF will result in considerable error on both equivalent permeability and per-cycle energy loss, which will be discussed in a later section of this work. To improve the accuracy, other PDFs could be adopted.

In the work of [14], the authors have developed an approach to numerically construct the PDF using experimentally measured symmetrical hysteresis loops, after discretising $p(U, V)$ with homogeneous grids on the $(U, V)$ plane and assuming that inside each grid $p(U, V)$ is constant. This approach is potentially able to capture the hysteresis of arbitrary materials including ferrites, but significant numerical error could be involved in practice, as has been discussed and improved in [15]. In order to achieve acceptable resolution, large number of hysteresis looped should be measured, which is in some cases impractical from engineering point of view. [16] has introduced a discrimination function in order to reduce the number of loops necessarily to be measured. Nevertheless, calculating the Everett integration of the discretised form of $p(U, V)$ in Preisach model could be too complicated and inefficient during time-domain simulation.

Authors of [17], [18] and [19] have explored that all the magnetisation curve of the Preisach model following the formulation in equation (1) can be derived from the descending curve of only one measured limiting hysteresis loop (with the highest amplitude of concern), without the need of analytically identifying the PDF. Making use of this feature, symmetrical hysteresis loops with relatively large amplitude (larger than $50 \%$ of the limiting loop) have been modelled with good accuracy in the work of [20], [21] and [22] for ferrite materials. Hysteresis loops with low amplitudes (e.g. $20 \%$ of the limiting loop), however, were not explicitly verified and no handling was specifically described to control the accuracy there. With desire to use Preisach model for arbitrary operation conditions, the accuracy control of minor hysteresis loops requires further improvement.

It has been discussed in [23] that reversible magnetisation is also present in soft magnetic materials which can not be captured by the classical Preisach model. Therefore the hysteresis model should be composed of a irreversible component using classical Preisach model and a reversible component.
The reversible component is essentially a single-lined $B-H$ characteristic, for which the inverse trigonometric (arctan) function proposed by [24] and hyperbolic cotangen (coth) function by [25], [26] have been adopted by [12] and [22], respectively. This work will still follow this methodology but a new fitting function is chosen for the reversible component, which provides additional degrees of freedom so that the equivalent permeability can be better controlled.

Aiming to improve the accuracy of simulating the hysteresis effect of ferrite materials in system-level time-domain simulation, this work combines the following aspects together, by which is distinguished from the other previous publications:

- Logistic probability distribution function is adopted for the irreversible component modelled by classical Preisach model, which is able to better approximate the percycle hysteresis energy loss of ferrite materials. The comparison to Cauchy-Lorentz PDF is provided in a later section.

- Improved parametrisation process is introduced for the irreversible component to control the accuracy of the percycle energy loss for both symmetrical large limiting- and small minor loops.

- A new form of reversible magnetisation curve is proposed, which gets the equivalent permeability of hysteresis loop at wide amplitude range under control.

- The improved modeling's approach is seamlessly incorporated into permeance-capacitor based magnetic circuit for system level simulations.

This paper is organised as follows: Section II provides the implementation details of the classical Preisach model in permeance-capacitor magnetic circuit, as an elaboration of the corresponding part from [3]. Section III demonstrates the proposed approach of modeling together with the procedure for parameter identification. Afterwards in Section IV, the fidelity of the model in simulating different ferrite materials is evaluated on a magnetic characterisation setup, together with the comparison to the Preisach model using CauchyLorentz PDF. Further in Section V the performance of the model in simulation environment for power electronic circuits is demonstrated and evaluated.

\section{Classical Preisach Model}

In this section, the basic concept of permeance-capacitor based magnetic circuit as well as its realisation of classical Preisach model for magnetic hysteresis is elaborated, as an extension of the corresponding part presented in [3]. The integrated magnetic structure depicted in Fig. 2a is taken as example, which is composed of two E-shape cores and four electrical windings. The magnetic structure resembling the real geometry can be intuitively translated into a permeance magnetic circuit shown in Fig. 2b. The interface components connecting electric- and magnetic circuit represent the windings (highlighted by the light blue frame), which are realised as gyrator form shown in Fig. 2c. Each permeance block stands for a certain part of the magnetic core (e.g. the one highlighted by the red frame in Fig. 2b represents one half of the middle limb in Fig. 2a). In the magnetic circuit, we have 


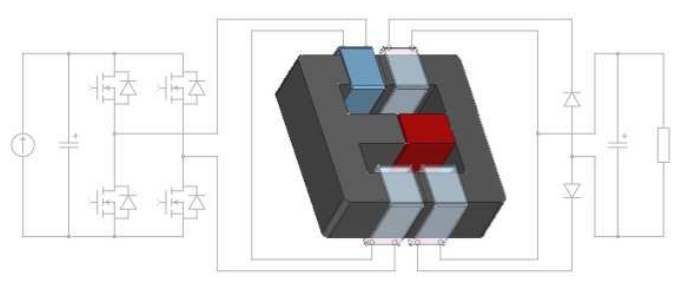

(a)

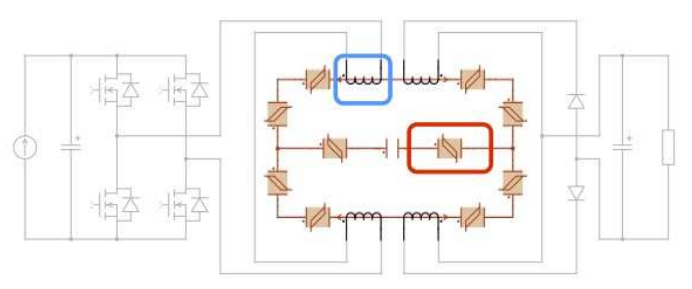

(b)

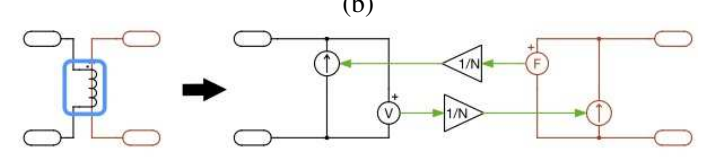

(c)

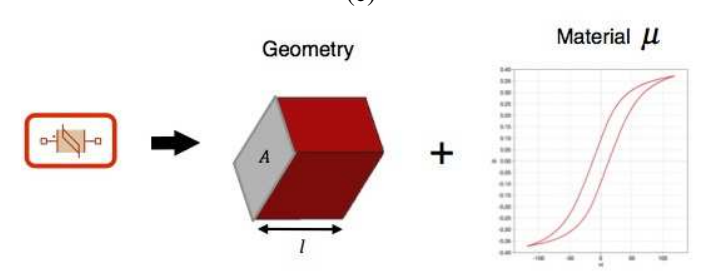

(d)

Fig. 2: Permeance-capacitor approach (a) Integrated magnetic component; (b) Magnetic circuit of the integrated magnetic component; (c) Winding component using gyrator structure as interface between electrical- and magnetic circuit; (d) Core block parametrised by geometry and material characteristic.

the combination of the through variable (the derivative of the magnetic flux $\dot{\Phi}$ ) and the across variable (magnetomotive force $F$ ). The relation between $\dot{\Phi}$ and $F$ on a single permeance block is governed by

$$
\dot{\Phi}=\mathscr{P} \cdot \frac{d F}{d t}
$$

the permeance value $P$ in the equation above is calculated using the geometry and material characteristic

$$
\mathscr{P}=\mu \cdot \frac{A}{l}
$$

where $A$ is the cross section area and $l$ is the magnetic path length. The $B-H$ characteristic of the material is reflected by the permeability $\mu$, as illustrated in Fig. $2 d$. Should the material nonlinearity (e.g. magnetic hysteresis) be considered, $\mu$ becomes a variable depending on the field strength $H$. The internal structure of a permeance block with hysteresis behaviour is demonstrated in Fig. 3. The magnetomotive force $F$ across the variable permeance component is measured and divided by the magnetic path length $l$, yields the field strength $H$. The classical Preisach model is described in C-Script which takes $H$ as well as the flux rate $\dot{\Phi}$ as input variables. The C-Script outputs the instantaneous differential permeability $\mu(H)=d B / d H$, which is further on multiplied by the geometry factor $A / l$ and provided to the variable permeance block. The calculation of $\mu(H)$ during simulation is introduced in below:

- Virgin curve: Assuming that the time-domain simulation initiates from the completely demagnetised state, the boundary between the positive- $\left(S_{+}\right)$and negative area $\left(S_{-}\right)$of the Preisach plane lays on $U=-V$, such that the flux density $B$ calculated using equation (1) is equal to 0 , as depicted on the right hand side of Fig. 4a. Under positive excitation (e.g. with positive voltage applied on the winding of the magnetic component so that $\dot{\Phi}>0$ ) the operation point on the $B-H$ plane moves along the virgin curve, the flux density $B$ is calculated as Everett integration following equation (1).

$$
\begin{aligned}
& B(H)=\iint_{S_{+}} p_{s}(U) \cdot p_{s}(-V) \cdot d U d V \\
&-\iint_{S_{-}} p_{s}(U) \cdot p_{s}(-V) \cdot d U d V
\end{aligned}
$$

On the $B-H$ plane, the instantaneous differential permeability $\mu$, or in other words, the slope of the $B-H$ curve, is graphically demonstrated on the left hand side of Fig. $4 \mathrm{a}$ and given as

$$
\mu_{\uparrow}(H)=2 \cdot p_{s}(H) \cdot \int_{-H}^{+H} p_{s}(-V) \cdot d V
$$

In the equation above, the integral term turns out to be closed-form if the probability distribution function $p_{s}$ can be analytically integrated, so as is the case of CauchyLorentz PDF which has been adopted in the existing publications. Thus $\mu$ with explicit expression can be directly substituted into equation (3) and assigned to the permeance core block of the magnetic circuit.

- Descending curve: Assuming that when $H$ reaches the positive peak (p.u. +1), the external excitation changes the polarity. Afterwards, the boundary between the areas $S_{+}$and $S_{-}$should move in the direction indicated on the right hand side of Fig. 4b. This polarity change is detected by the C-Script via examing the sign of the second input $\dot{\Phi}$ (time derivative of the flux $\Phi$ ) and the first field strength

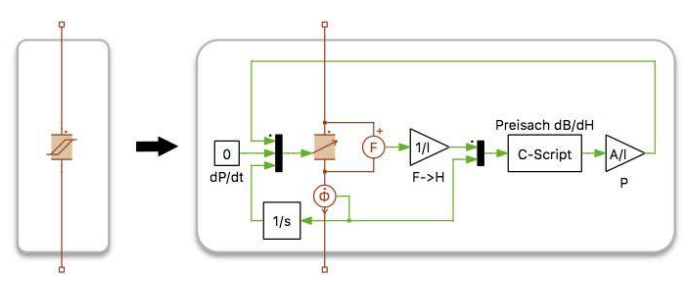

Fig. 3: Structure of the permeance block with hysteresis behaviour using classical Preisach model. 


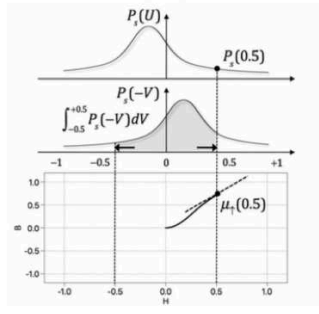

(a)

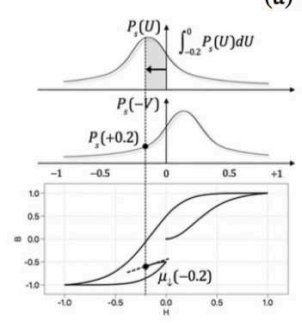

(d)
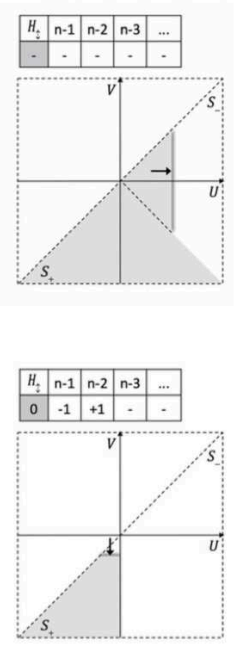$$
\text { d) }
$$

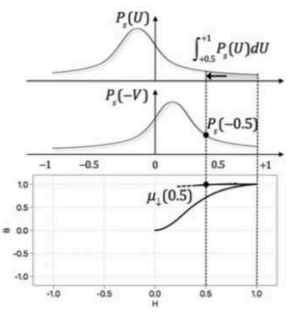

(b)

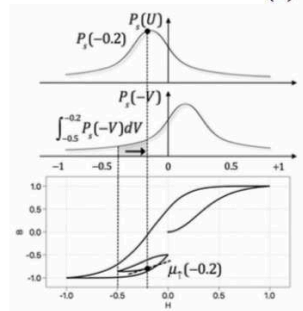

(e)
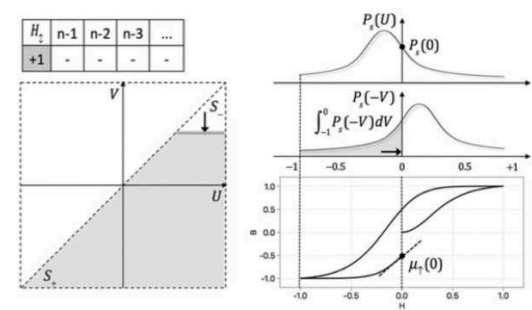

(c)
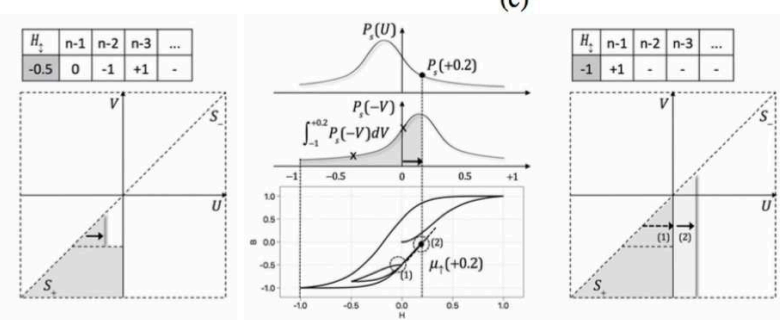

(f)

Fig. 4: Calculation of the permeability and the area separation of Everett integral (a) on the virgin curve; (b) on the descending curve after reaching the positive peak; (c) on the ascending curve after reaching the negative peak; (d) on the descending curve in an asymmetrical minor loop; (e) on the ascending curve in an asymmetrical minor loop; (f) on the ascending curve after exiting the minor loop.

extremity +1 is pushed into a stack structure as shown on the top-right of Fig. $4 \mathrm{~b}$. From this time point on, $\mu$ is calculated using the equation below with extremity $H_{\uparrow}=$ +1 .

$$
\mu_{\downarrow}(H)=2 \cdot p_{s}(-H) \cdot \int_{H}^{H_{\uparrow}} p_{s}(U) \cdot d U
$$

- Ascending curve: The external excitation becomes positive after $H$ reaches the negative peak (p.u. -1) as shown in Fig. 4c. The negative peak is pressed into the stack of extremities and the actual $H_{\uparrow}$ becomes -1 , meanwhile the previous extremity +1 is shifted to a deeper level. The permeability on the ascending curve is given as

$$
\mu_{\uparrow}(H)=2 \cdot p_{s}(H) \cdot \int_{H_{\downarrow}}^{H} p_{s}(-V) \cdot d V
$$

- Minor loop: The external excitation switches its polarity again to negative at $H=0$ on the ascending curve, so that a minor hysteresis loop is initiated (Fig. 4d). At the very moment, $H_{\uparrow}$ becomes 0 while the previous two extremities -1 and +1 are shifted one level deeper in the stack. The permeability on the descending curve of the minor loop has the same form as equation (6), except for the integral limit $H_{\uparrow}=0$. Afterwards, let's assume that the ascending curve of the minor loop begins at $H=-0.5$ where $H_{\uparrow}$ becomes -0.5 (Fig. 4e). Before $H$ reaches the over last extremity $(H=0)$ there are four values in total $(-0.5,0,-1,+1)$ stored in the stack, and $\mu$ is retained following equation (7) with $H_{\uparrow}=-0.5$.

- Exit from Minor loop: At the moment when $H$ just tends to exceed the over last extremity $(H=0)$, the operation point is exactly located on the position where the minor loop was initiated previously (point (1) in Fig. 4f). At this time point, the latest two extremities -0.5 and 0 are erased from the stack. From then on, the operation point travels along the ascending curve with $H_{\uparrow}=-1$, as if the minor loop has never happens (point (2) in Fig. 4f). This deletion property of Preisach model corresponds to the characteristic of ferrite material in reality, which will be verified by the experimental result in a later section of this work.

\section{PRoposed MODEL}

The main objective of the proposed modeling approach is to achieve good accuracy of simulating magnetic hysteresis in a wide range of amplitudes (both large major- and small minor loops), which is evaluated by the two criterias below:

- Per cycle energy loss: The area enclosed by the hysteresis loops on the $B-H$ plane.

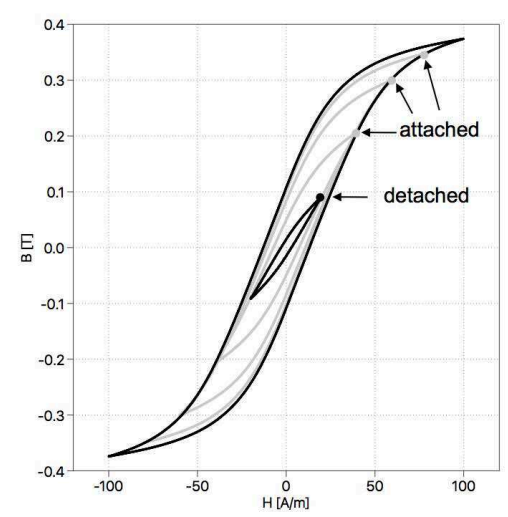

Fig. 5: Measured hysteresis loops of ferrite material N87 from $\mathrm{H}=100 \mathrm{~A} / \mathrm{m}$ to $\mathrm{H}=20 \mathrm{~A} / \mathrm{m}$ at $200 \mathrm{~Hz}$. 


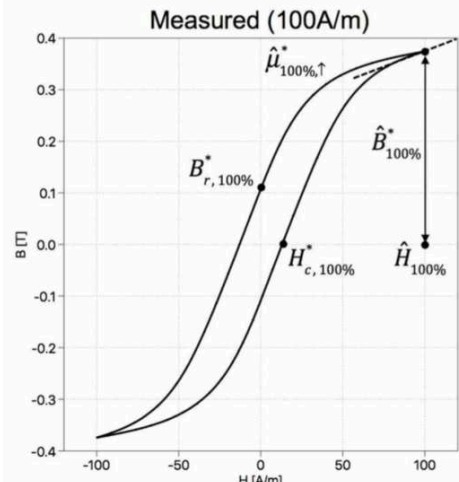

(a)

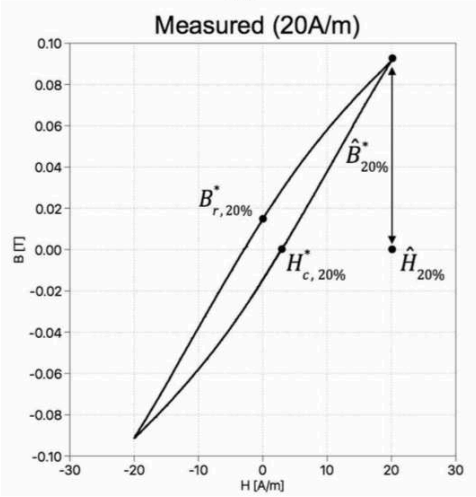

(c)
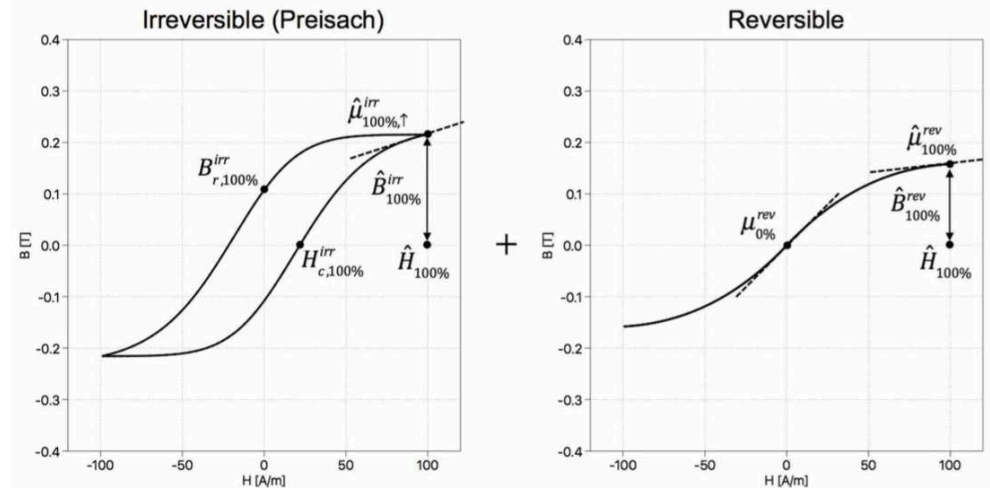

(b)
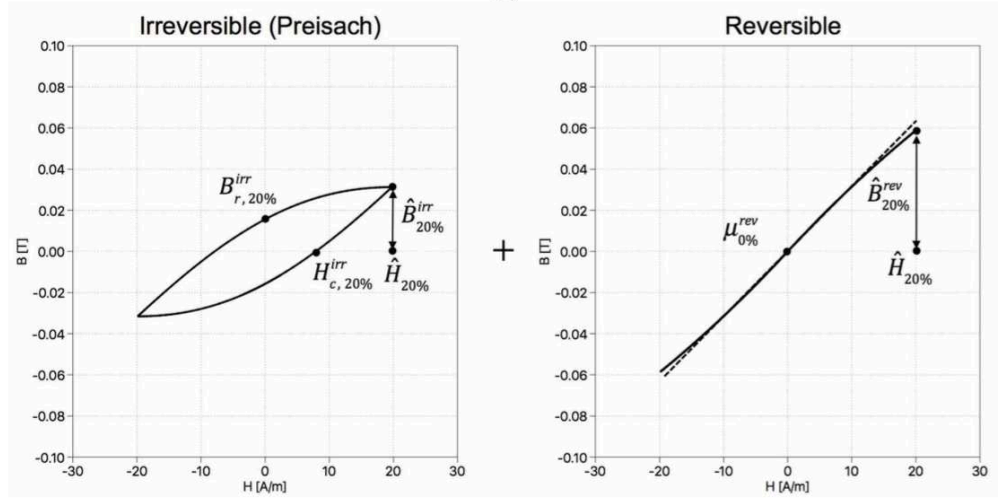

(d)

Fig. 6: a) Measured limiting hysteresis loop with amplitude $H_{100 \%}=100 \mathrm{~A} / \mathrm{m}$; (b) Hysteresis model composed of irreversibleand reversible component with amplitude $H_{100 \%}=100 \mathrm{~A} / \mathrm{m}$; (c) Measured limiting hysteresis loop with amplitude $H_{20 \%}=$ $20 \% \cdot H_{100 \%}=20 \mathrm{~A} / \mathrm{m}$; (b) Hysteresis model composed of irreversible- and reversible component with amplitude $H_{20 \%}=$ $20 \% \cdot H_{100 \%}=20 \mathrm{~A} / \mathrm{m}$.

- Equivalent inductivity: Slope of the virtual straight line connecting the positive- and negative peaks.

For illustration purpose, the characteristic of ferrite material $\mathrm{N} 87$ experimentally measured at $200 \mathrm{~Hz}$ is taken as an example. In Fig. 5 the hysteresis loops of different amplitudes are displayed together. We define the one with the largest field strength amplitude $\hat{H}_{100 \%}$ (e.g. $100 \mathrm{~A} / \mathrm{m}$ ), which makes the flux density approach saturation, as the "limiting loop". The "limiting loop" indicates the valid operation range of the model and it is assumed that the hysteresis effect is of concern only inside this range within the simulation. Inside the the limiting loop, one can realise that peaks of the other loops with relatively large amplitudes $40 \% \cdot \hat{H}_{100 \%} \sim 80 \% \cdot \hat{H}_{100 \%}(40 \mathrm{~A} / \mathrm{m}$ $\sim 80 \mathrm{~A} / \mathrm{m}$ ) almost attach on the lower boundary of the limiting loop. This phenomenon has been also observed from other ferrite materials, which indicates that if a simulation model is able to reproduce the lower boundary of the limiting loop, the smaller ones can be also well presented, at least in terms of the equivalent inductivity. However this theory does not apply to the minor hysteresis loop with very low amplitude, say $20 \% \cdot \hat{H}_{100 \%}(20 \mathrm{~A} / \mathrm{m})$, whose peaks obviously deviate from the lower boundary of the limiting loop.

In the approach proposed in this work, only one limiting hysteresis loop (Fig. 6a) and one symmetrical minor hysteresis loop (Fig. 6c) are required to be experimentally measured as input, and the model is parametrised to approximate these two loops. We suppose the hysteresis loops with amplitudes between the measured limiting- and minor loops can be automatically fitted thanks to the physical-based intrinsic property of the Preisach model, which will be verified in a later section via experimental tests.

Following the method proposed by [23], in this work we also construct the hysteresis model as the summation of an irreversible- and a reversible component, which is graphically demonstrated in Fig. 6b and Fig. 6d for the limiting loop and the minor loop, respectively. Please note that the same classical Preisach model is applied for the irreversible component, and the reversible component in Fig. $6 \mathrm{~d}$ is just one part of the same curve in Fig. 6b, within the field strength range $\left[-H_{20 \%},+H_{20 \%}\right]$. The formulation and parameter identification of the two component is introduced in the following subsections.

\section{A. Determination of the irreversible component}

Contrary to the existing publications, the "Logistic" probability distribution function is adopted for the irreversible component, which is represented by the classical Preisach model. The generalised form of the logistic PDF can be expressed as 


$$
p_{s}(H)=K \cdot \frac{e^{-\left(H-H_{0}\right) \cdot \sigma}}{\left(1+e^{-\left(H-H_{0}\right) \cdot \sigma}\right)^{2}}
$$

where $K, H_{0}$ and $\sigma$ are parameters to be determined. Similar to the Cauchy-Lorentz PDF which has been chosen by the existing publications, the logistic PDF can be integrated analytically, thus the cumulative distribution function (CDF) of logistic PDF is given as

$$
C_{s}(H)=\frac{K \cdot \sigma}{1+e^{-\left(H-H_{0}\right) \cdot \sigma}}
$$

In this way, the permeability $\mu$ can be calculated in a closedform (Equation (5) (7)). Due to the fact that the reversible component has zero remanence, the remanent flux density of the simulated irreversible component $\left(B_{r, 100 \%}^{i r r}\right.$ in Fig. $6 \mathrm{~b}$ and $B_{r, 20 \%}^{i r r}$ in Fig. 6d) must be equal to that of the measurement.

$$
\text { Conditions }\left\{\begin{array}{l}
B_{r, 100 \%}^{i r r} \triangleq B_{r, 100 \%}^{*} \\
B_{r, 20 \%}^{i r r} \triangleq B_{r, 20 \%}^{*}
\end{array}\right.
$$

where $B_{r, 100 \%}^{*}$ and $B_{r, 20 \%}^{*}$ are the remanent flux density of the measured limiting- and minor loops, as has been highlighted in Fig. 6a and Fig. 6c, and any values from experimental measurement in this work are denoted with superscript "*". As has been explored by the authors of [27], the remanence flux density of Preisach using independent PDF can be explicitly expressed by

$$
\begin{array}{r}
B_{r, 100 \%}^{i r r}=\int_{0}^{\hat{H}_{100 \%}} p_{s}(U) d U \int_{-\hat{H}_{100 \%}}^{0} p_{s}(-V) d V \\
=\left(C_{s}\left(\hat{H}_{100 \%}\right)-C_{s}(0)\right)^{2} \triangleq B_{r, 100 \%}^{*}
\end{array}
$$

the equation above is also valid for the symmetrical minor loop

$$
\begin{aligned}
B_{r, 20 \%}^{i r r}=\int_{0}^{\hat{H}_{20 \%}} & p_{s}(U) d U \int_{-\hat{H}_{20 \%}}^{0} p_{s}(-V) d V \\
& =\left(C_{s}\left(\hat{H}_{20 \%}\right)-C_{s}(0)\right)^{2} \triangleq B_{r, 20 \%}^{*}
\end{aligned}
$$

Apart from the metal-based materials, since ferrite materials do not undertake field-annealing treatment during the manufacture process, it is reasonable to assume symmetrical nature for the PDF of classical Preisach model. Therefore the bias parameter $H_{0}$ of the irreversible part (equation (8) and (9)) is chosen to be 0 in this work, which makes the PDF be symmetrical about the vertical axis. In this way, the remaining two parameters $K$ and $\sigma$ can be fully determined via solving the two equations (11) and (25). Due to nonlinearity, commonly used iteration method like Newton-Raphson can be adopted. Please note that the per-cycle energy loss is controlled by the irreversible component.

\section{B. Determination of the reversible component}

After the Preisach model parameters of the irreversible component (classical Preisach model) are identified, the reversible component is parametrised to make the summation of the two components with the same peak point as the measured limiting- and minor loop, or in other words, to control the equivalent permeability of them. Instead of arctan and coth proposed by the existing publications, a new form of "Sigmoid" function is proposed in this work, in order to shape the reversible component with more degrees of freedom. The reversible $B^{r e v}(H)$ is constructed as the integration of a shifted arctan function, and the flux density when $H \geq 0$ is governed by the equation below.

$$
\begin{gathered}
B^{r e v}(H)=\int_{0}^{H}\left(F \cdot \arctan \left(\left(H_{1}-x\right) \cdot \alpha\right)+D\right) d x \\
=-F / \alpha \cdot\left(\left(H_{1}-H\right) \cdot \alpha \cdot \operatorname{atan}\left(\left(H_{1}-H\right) \cdot \alpha\right)\right. \\
\left.-0.5 \ln \left(1+\left(H_{1}-H\right)^{2} \cdot \alpha^{2}\right)\right)+F \alpha \cdot\left(H_{1} \cdot \alpha \cdot \operatorname{atan}\left(H_{1} \cdot \alpha\right)\right. \\
\left.+0.5 \ln \left(1+H_{1}^{2} \cdot \alpha^{2}\right)\right)+D \cdot H
\end{gathered}
$$

The permeability $\mu^{r e v}(H)$ to be provided to the permeance block in the magnetic circuit is the derivative of equation (13) about field strength $H$, or in other words, the shifted arctan function itself.

$$
\mu^{r e v}(H)=F \cdot \arctan \left(\left(H_{1}-H\right) \cdot \alpha\right)+D
$$

In the operation range with field strength $H<0$, the mirror of equations (13) and (14) about the origin of the $B-H$ plane are applied. The parameter $\alpha$ is preliminary configured to be 0.01 and can be adjusted to improve the permeability accuracy, which will be described later in sub-section III-C. The identification of the other three parameters $H_{1}, F$ and $D$ is described in the following:

In the Preisach model, the symmetry of the irreversible component's PDF (due to the parameter configuration $H_{0}=0$ ) leads to the fact that the peak flux density $\hat{B}_{100 \%}^{i r r}$ of the simulated limiting loop is two times of the remanent flux density $B_{r, 100 \%}^{i r r}$ (equal to $B_{r, 100 \%}^{*}$ ) after the parameter identification described in section III-A.

$$
\hat{B}_{100 \%}^{i r r}=2 \cdot B_{r, 100 \%} \triangleq 2 \cdot B_{r, 100 \%}^{*}
$$

Therefore the peak flux density of the reversible component at $\hat{H}_{100 \%}$ can be extracted from the measured peak flux density of the limiting loop, given by

$$
\hat{B}_{100 \%}^{r e v}=B_{100 \%}^{*}-\hat{B}_{100 \%}^{i r r}=B_{100 \%}^{*}-2 \cdot B_{r, 100 \%}^{*}
$$

As has been observed at the beginning of section III, the peak of the relatively large minor loops attach the lower boundary of the limiting loop. In order to make the simulated lower boundary of the limiting loop close to that from the measurement, the permeability of the reversible component at $\hat{H}_{100 \%}\left(\hat{\mu}_{100 \%}^{r e v}\right.$ in Fig. 6b) should be controlled as well, which is given by subtracting the permeability of the irreversible component model from the measured one: 


$$
\begin{aligned}
& \hat{\mu}_{100 \%}^{r e v}=\hat{\mu}_{100 \%}^{*}-\hat{\mu}_{100 \%}^{i r r} \\
& =\hat{\mu}_{100 \%}^{*}-p_{s}\left(\hat{H}_{100 \%}\right)\left(C_{s}\left(\hat{H}_{100 \%}\right)-C_{s}\left(-\hat{H}_{100 \%}\right)\right)
\end{aligned}
$$

In the case of the minor symmetrical loop with field strength amplitude $\hat{H}_{20 \%}=20 \% \cdot \hat{H}_{100 \%}$, the irreversible component described by Preisach model is calculated using solely the part of the PDF inside the range $\left[-\hat{H}_{20 \%},+\hat{H}_{20 \%}\right]$. With the previous assumption $H_{0}=0$, this part of PDF is also symmetrical about origin so that the relation described in equation (15) is valid here as well.

$$
\hat{B}_{20 \%}^{i r r}=2 \cdot B_{r, 20 \%} \triangleq 2 \cdot B_{r, 20 \%}^{*}
$$

If the reversible component can be considered as a linear function close to origin as depicted in Fig. 6d, the initial permeability of the reversible component can be obtained via subtracting $\hat{B}_{20 \%}^{i r r}$ from the peak flux density of the measured minor loop and dividing by $\hat{H}_{20 \%}$.

$\mu_{0 \%}^{r e v}=\left(\hat{B}_{20 \%}-\hat{B}_{20 \%}^{i r r}\right) / \hat{H}_{20 \%}=\left(\hat{B}_{20 \%}^{*}-2 \cdot B_{r, 20 \%}^{*}\right) / \hat{H}_{20 \%}$

Up to this stage, with the parameter $\alpha$ given, the other three parameters $H_{1}, F$ and $D$ of the reversible component can be determined via solving the three equations (16), (17) and (19), substituting $\hat{B}_{100 \%}^{r e v}, \hat{\mu}_{100 \%}^{r e v}$ and $\mu_{0 \%}^{r e v}$ by the expression from equations (13) and (14). Newton-Raphson iteration is adopted for the parameter identification and initial values should be configured to guarantee the convergency.

$$
\left\{\begin{array}{l}
H_{1}^{(0)}=\frac{\hat{B}_{100 \%}^{r e v}-\hat{H}_{100 \%} \cdot \hat{\mu}_{100 \%}^{r e v}}{\mu_{0 \%}^{r e v}-\hat{\mu}_{100 \%}^{r e v}} \\
K^{(0)}=\frac{\mu_{0 \%}^{r e v}-\hat{\mu}_{100 \%}^{r e v}}{v_{1}-v_{2}} \\
D^{(0)}=\frac{\mu_{0 \%}^{r e v} \cdot v_{2}-\hat{\mu}_{100 \%}^{r e v} \cdot v_{1}}{v_{2}-v_{1}}
\end{array}\right.
$$

where $\hat{B}_{100 \%}^{r e v}, \hat{\mu}_{100 \%}^{r e v}$ and $\mu_{0 \%}^{r e v}$ are given by the right hand side of equations (16), (17) and (19), respectively, while $v_{1}$ and $v_{2}$ are provided in below.

$$
\begin{gathered}
v_{1}=\arctan \left(H_{1}^{(0)} \cdot \alpha\right) \\
v_{2}=\arctan \left(\left(H_{1}^{(0)}-\hat{H}_{100 \%}\right) \cdot \alpha\right)
\end{gathered}
$$

\section{Final adjustment}

For the sake of further controlling the simulated lower boundary of the limiting loop to approach that from the measurement, which intrinsically determines the peak point of the other minor loops with amplitude close to the limiting loop (as has been discussed in Fig. 5), the parameter $\alpha$ can be adjusted.

The goal is to make the simulated flux density $B_{50 \%}$ at $H_{50 \%}=0.5 \cdot \hat{H}_{100 \%}$ be equal to that on the limiting loop's ascending curve. The parameter $\alpha$ determines the curvature

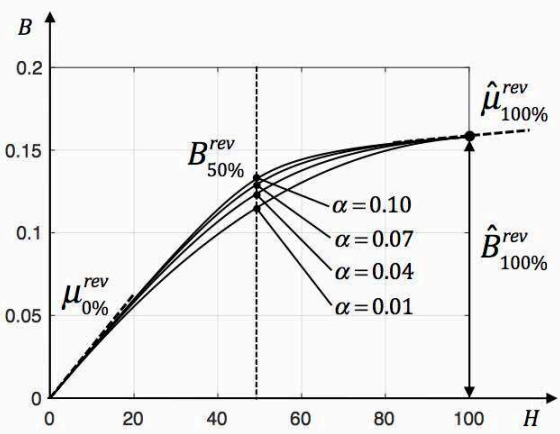

(a)

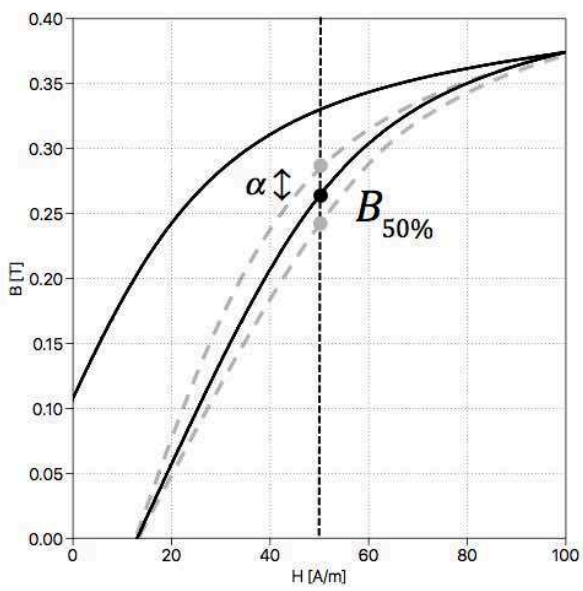

(b)

Fig. 7: (a) Influence of the model parameter $\alpha$ on the curvature of the irreversible component; (b) Influence of the model parameter $\alpha$ on the lower boundary of the limiting hysteresis loop after summing up the reversible- and irreversible component together.

of the reversible component such that the flux density $B_{50 \%}^{r e v}$ at $H_{50 \%}$ on the reversible component can be adjusted via changing $\alpha$, as demonstrated in Fig. 7a. In Fig. 7a, the NewtonRaphson iteration described in the section III-B should be carried out for any adopted $\alpha$ value, so that the point $\hat{B}_{100 \%}^{r e v}$ as well as the slopes $\hat{\mu}_{100 \%}^{r e v}$ and $\mu_{0 \%}^{r e v}$ remains the same. Please note that at this stage, the irreversible component has been completely determined and its parameter remains unchanged, which leads to the fact that the change of $B_{50 \%}^{\text {rev }}$ due to adjustment of $\alpha$ is directly reflected on $B_{50 \%}$ which locates on the lower boundary of the simulated limiting loop, as shown in Fig. 7b.

For parametrisation of the parameter $\alpha$, the direct objective is to have the reversible flux density $B_{50 \%}^{r e v}$ at $H_{50 \%}$ (obtained from equation (13)) plus the peak flux density $\hat{B}_{50 \%}^{i r r}$ of the simulated irreversible hysteresis loop with field strength amplitude $H_{50 \%}$ be equal to the flux density $B_{50 \%}^{*}$ at $H_{50 \%}$ on the ascending branch of the measured limiting loop:

$$
B_{50 \%}^{r e v}+\hat{B}_{50 \%}^{i r r} \triangleq B_{50 \%}^{*}
$$

Since the relationship between the remanence- and peak flux density presented in equations (15) and (18) is also valid for 


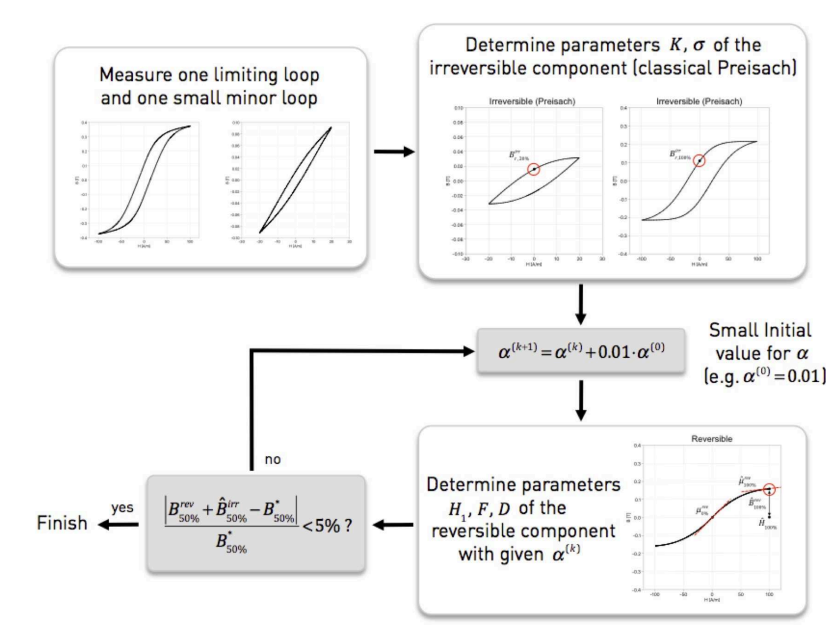

Fig. 8: Parametrisation of the proposed model including the iteration to determine the parameter $\alpha$.

the irreversible hysteresis loop with field strength amplitude $H_{50 \%}$, the peak flux density $\hat{B}_{50 \%}^{i r r}$ from equation (23) is given by

$$
\hat{B}_{50 \%}^{i r r}=2 \cdot B_{r, 50 \%}
$$

following the same formulation from equations (11) and (25), $B_{r, 50 \%}$ can be explicitly expressed as

$$
B_{r, 50 \%}=\left(C_{s}\left(\hat{H}_{50 \%}\right)-C_{s}(0)\right)^{2}
$$

Thanks to the fact that $\alpha$ is monotonously related to $B_{50 \%}^{r e v}$ ( $B_{50 \%}^{r e v}$ increases with higher $\alpha$ ), the adjustment of $\alpha$ can be easily conducted either manually or automatically via adding another fitting loop on the top of the Newton-Rahphson iteration introduced in section III-B, as illustrated in Fig. 8: After the parameters of the irreversible component are determined (subsection III-A), a small initial value is configured to the parameter $\alpha$ (e.g. $\alpha^{(0)=0.01)}$ so that the left hand side of equation (23) is lower than the measured $B_{50 \%}^{*}$. Then a simple iterative process is started, in each cycle $\alpha$ is added up with a small increment of $0.01 \cdot \alpha^{(0)}$, and the parameters $H_{1}, F, D$ are obtained with the new $\alpha$ using Newton-Raphson iteration (subsection III-B). At the end of each cycle, the left hand side of equation (24) is evaluated and compared to the measured $B_{50 \%}^{*}$. The iteration is terminated as soon as the error becomes lower than $5 \%$.

\section{Model structure}

The structure of the proposed combined hysteresis model in a permeance-capacitance based magnetic circuit is shown in Fig. 9. Two variable core blocks are connected in parallel, to account for the irreversible- and reversible component, respectively. The permeance value of the two core blocks are calculated in two separate C-Scripts and scaled with the geometric coefficient $A / l$. The equivalent permeance of the whole hysteresis core block is essentially the sum of the individual permeances, which are provided to the first input of the variable core blocks.

$$
\mathscr{P}(H)=\mu^{i r r}(H) \cdot \frac{A}{l}+\mu^{r e v}(H) \cdot \frac{A}{l}
$$

Since differential permeability has been used throughout this work, the second input of the variable core blocks $d \mathscr{P} / d t$ can be provided by a constant zero, as has been discussed in [3]. The third input of the variable core blocks accepts the flux density of the irreversible- and reversible components, which are obtained from integral of the flux rate $\dot{\Phi}$, in order to make the simulation solver hold Kirchhoff's junction law for the magnetic circuit.

\section{VERIFICATION IN CONTINUOUS CIRCUIT}

For validation of the proposed modeling approach, a test bench based on the two winding approach described in [28] has been established to measure the hysteresis loop of the core materials, as shown in Fig. 10a and Fig. 10b. Since geometry impact is not in scope of this work, only toroidal core is considered which is sufficiently representative for the material's characteristic. The toroidal core sample is equipped with two windings, the primary winding is supplied by a RF power amplifier (type LM3886, gain-bandwidth product $2 \mathrm{MHz}$ ) which generates sinusoidal voltage excitation. A shunt resistor together with its instrumentation amplifier circuitry is installed on the primary side to measure the current $I$, which is converted into field strength using the equation below:

$$
H=\frac{I \cdot N_{1}}{l}
$$

where $l$ is the equivalent magnetic path length of the core sample and $N_{1}$ the turns number of the primary winding. The secondary winding is left open and the voltage is measured using a resistor divider. The measured voltage is integrated to obtain the flux density, given by

$$
B=\frac{1}{A \cdot N_{2}} \int U d t
$$

where $A$ is the equivalent cross section area of the core sample, and $N_{2}$ the turns number of the secondary winding. A control unit (PLECS RT-Box 1) is connected to the test bench to generate reference signal for power amplifier and process the measurements. As this work focus on the frequency independent hysteresis effect without eddy current and residual effects, the power amplifier's output voltage has been configured as low frequency $200 \mathrm{~Hz}$ sinusoidal wave. As the

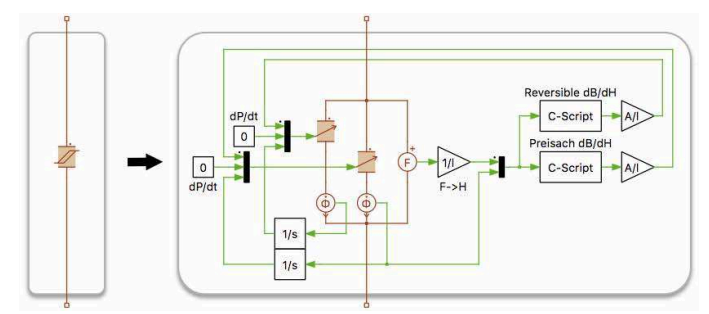

Fig. 9: Model structure of the permeance block using proposed model. 
temperature dependency is also not in the scope of this work, all tests have been conducted under room temperature of $25^{\circ} \mathrm{C}$.

The simulation model (top of Fig. 10b) is established in the system-level simulation software PLECS for power electronics. The hysteresis core block represents the core sample, whose geometric parameters $A$ and $l$ are configured using the values from the datasheet, while the material characteristic is modelled and parametrised using the approach introduced in section III. The power amplifier is modelled as an ideal sinusoidal AC voltage source, in series to which the equivalent resistance (including the shunt resistor for current measurement and parasitics, measured in DC condition) is connected. All components are configured to match the test bench hardware.

The ferrite material N87 from TDK is taken as the first verification case, where the toroidal core of size code " $R$ $41.8 \times 26.2 \times 12.5$ " is taken as sample, the turns number of primary and secondary windings are both eight. As input of the parameter identification process which is introduced in the section III, the limiting hysteresis loop with amplitude $\hat{H}_{100 \%}=100 \mathrm{~A} / \mathrm{m}$ and a symmetrical minor loop with amplitude $\hat{H}_{20 \%}$ are measured. Please note that the parameters of the model will remain the same in the other verification cases. In Fig. 11a and Fig. 11b, the simulated hysteresis loop as well

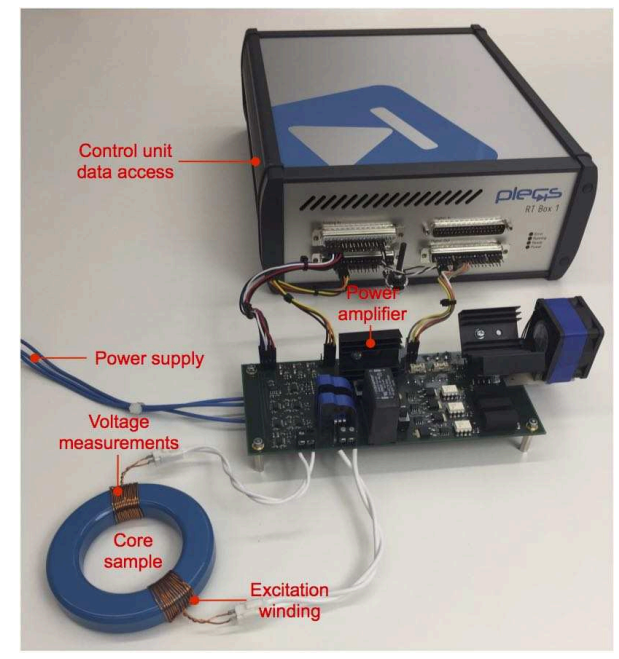

(a)
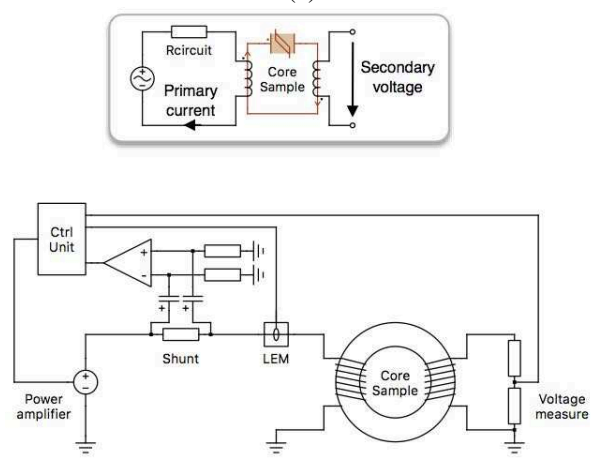

(b)

Fig. 10: (a) Hardware configuration of the test setup; (b) Schematic and simulation model of the test setup. as the time domain waveform of the primary winding current and secondary winding voltage at different field strength amplitudes $(20 \mathrm{~A} / \mathrm{m} \sim 100 \mathrm{~A} / \mathrm{m})$ are compared to the experimental measurement. Although an ideal sinusoidal voltage is generated on the power amplifier output, the measured primary winding current is heavily distorted, due to the presence of nonlinear hysteresis effect. The proposed simulation model is able to approximate the hysteresis loop on the $\mathrm{B}-\mathrm{H}$ plane as well as the time domain waveform well. Especially on the secondary voltage waveform in Fig. $22 \mathrm{~b}$, due to voltage drop on the circuit resistance as a coupled effect from the distorted circuit current, the secondary open-circuit voltage

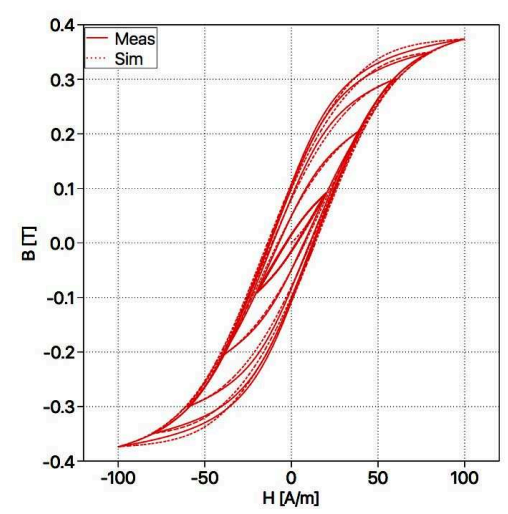

(a)
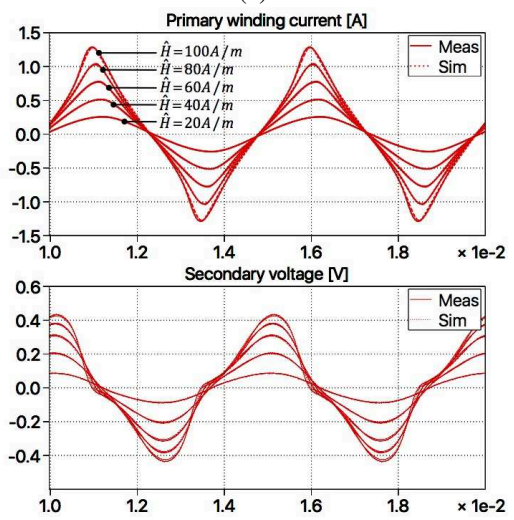

(b)

Fig. 11: Comparison between measurement and simulation of Ferrite N87 at different field strength amplitudes (a) Hysteresis loop; (b) Time-domain primary winding current and secondary voltage.

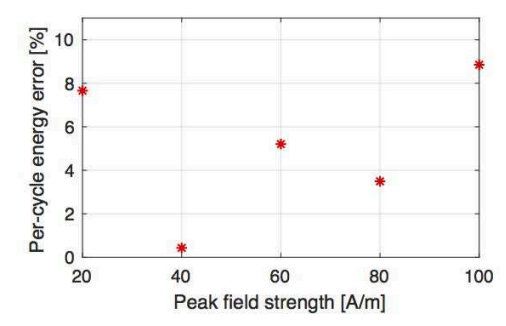

Fig. 12: Percentage error of the simulated per-cycle energy loss from simulation of ferrite N87 


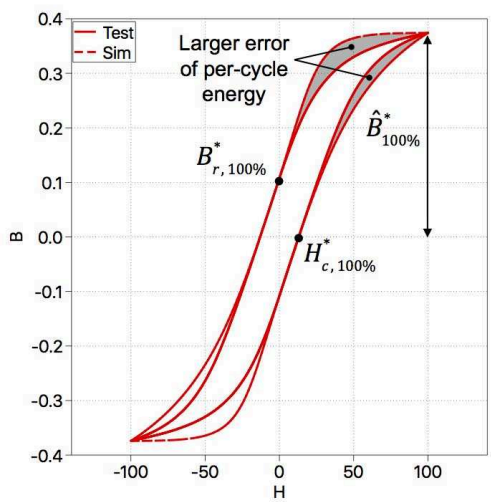

Fig. 13: Comparison of the limiting hysteresis loop between measurement and simulation using only classical Preisach model without the reversible component

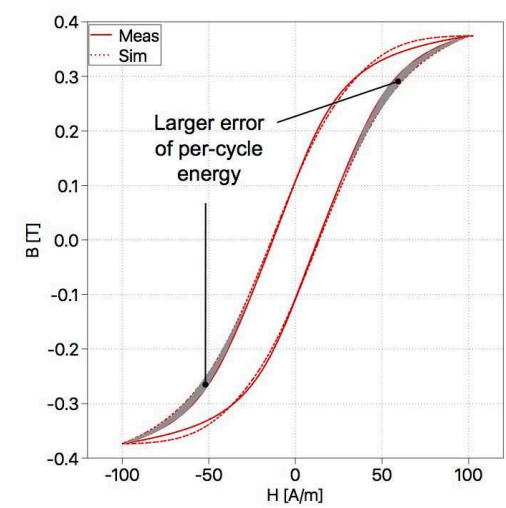

(a)

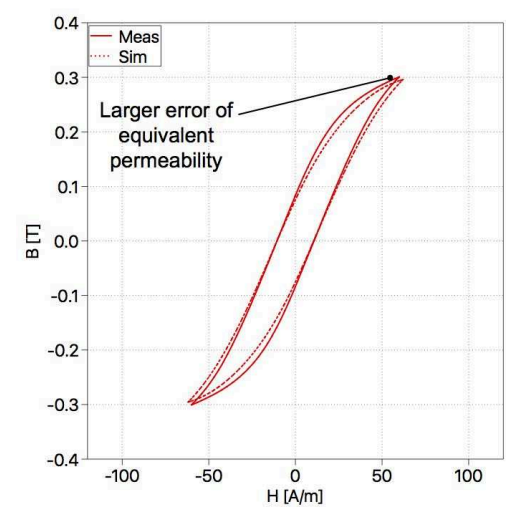

(b)

Fig. 14: Comparison between measurement and simulation of Ferrite N87 using Cauchy-Lorentz PDF (a) Hysteresis loop at $\hat{H}_{100 \%}=100 \mathrm{~A} / \mathrm{m}$; (b) Hysteresis loop at $\hat{H}_{60 \%}=60 \mathrm{~A} / \mathrm{m}$.

also includes harmonic component, which is captured by the model as well. In Fig. 11a, the peak point of the hysteresis loops nearly overlaps with the measurement, so that the error of the equivalent permeability is maintained under $1 \%$. The per-cycle energy loss has been measured and simulated via integrating the product of time domain voltage and current waveform for one AC period (equivalent to the enclosed area

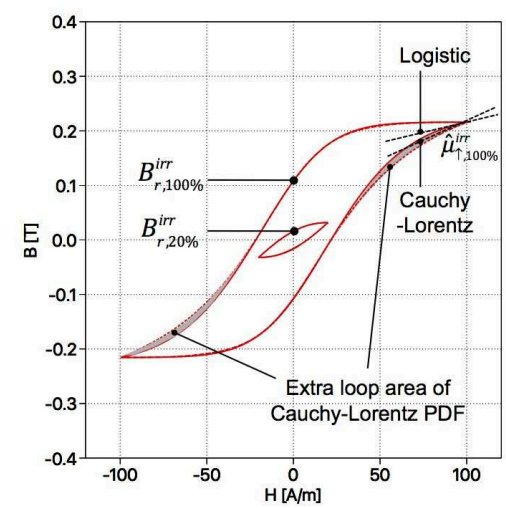

(a)

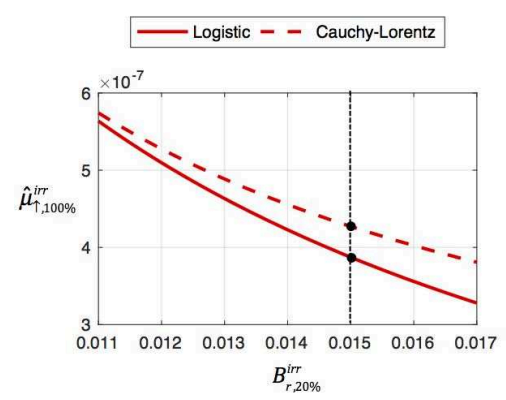

(b)

Fig. 15: Comparison of irreversible component between Preisach model using two different PDFs (a) Irreversible component with field strength amplitude $\hat{H}_{100 \%}=100 \mathrm{~A} / \mathrm{m}$; (b) Permeability on the top of the ascending branch of a limiting loop with model parameters fitted to fixed $B_{r, 100 \%}^{i r r}$ and varying $B_{r, 20 \%}^{i r r}$

of the $B-H$ loop), whose error between simulation and measurement are listed in Fig. 12. The maximum error $8.9 \%$ is present at the limiting hysteresis loop of high amplitude $\hat{H}_{100 \%}=100 \mathrm{~A} / \mathrm{m}$, while the error at the other operation points are all controlled below $10 \%$.

For comparison purpose, only the irreversible classical Preisach model using Cauchy-Lorentz PDF (shown in Fig. 3 ) is parametrised to approximate the peak flux density $\hat{B}_{100 \%}^{*}$, remanence flux density $B_{r, 100 \%}^{*}$ and the coercitive field strength $H_{c, 100 \%}^{*}$ of the measured limiting loop, following the method introduced in the work of [3]. The simulated $B-H$ characteristic exhibits significant larger loop area compared to the measurement, which result in much higher per-cycle energy loss (error over 30\%), as demonstrated in Fig. 13.

With the proposed reversible component included, if the irreversible component is calculated using Cauchy-Lorentz PDF (adopted by [12]) whose parameters are identified following the same procedure described in section III-A, the hysteresis loops with field strength amplitude of $100 \mathrm{~A} / \mathrm{m}$ (limiting loop) and $60 \mathrm{~A} / \mathrm{m}$ are compared to the measurement in Fig. 14a and Fig. 14b, respectively:

In Fig. 14a, the simulated limiting loop (at $\hat{H}_{100 \%}=$ $100 \mathrm{~A} / \mathrm{m}$ ) using Cauchy-Lorentz PDF exhibits significantly higher per-cycle energy loss, in comparison to the one using 
Logistic PDF (Fig. 11a). The excessive energy loss is indicated by the highlighted loop area difference (grey color). The error compared to the measurement is $11.5 \%$, which is higher than that obtained using Logistic PDF (8.9\%). The reason can be ascribed to the simulated differential permeability on the top of a ascending branch. The simulated irreversible component of the limiting loop (that of the right-hand side variable permeance block in Fig. 9) are compared between the models using Logistic- and Cauchy-Lorentz PDF in Fig. 15a, higher permeability $\hat{\mu}_{\uparrow, 100 \%}^{i r r}$ is present on the model using CauchyLorentz PDF, which leads to large enclosed area on the B-H plane and thus higher per-cycle energy loss.

One should recall that in the parameter identification procedure from section III-A the irreversible component is configured to approximate the remanent flux density of the limiting loop and that of a low amplitude minor loop, so that $B_{r, 100 \%}^{i r r}=B_{r, 100 \%}^{*}$ and $B_{r, 20 \%}^{i r r}=B_{r, 20 \%}^{*}$. To generalise the statement that $\hat{\mu}_{\uparrow, 100 \%}^{i r r}$ using Cauchy-Lorentz PDF is higher than that using Logistic PDF, we fit the parameters of the irreversible component such that the remanent flux density of the limiting loop $B_{r, 100 \%}^{i r r}$ is still equal to the measurement, while that of the low amplitude minor loop $B_{r, 20 \%}^{i r r}$ is fitted targeting different values. The resulted permeability $\hat{\mu}_{\uparrow, 100 \%}^{i r r}$ from the model using two PDFs are compared in Fig. 15b, independent of $B_{r, 20 \%}^{i r r}$, the permeability $\hat{\mu}_{\uparrow, 100 \%}^{i r r}$ from the model using Cauchy-Lorentz PDF is always higher than that using Logistic PDF, which leads to higher per-cycle energy loss in simulation.

Moreover in the case of N87 material, since the irreversible permeability $\hat{\mu}_{\uparrow, 100 \%}^{\text {irr }}$ calculated using Cauchy-Lorentz PDF is already higher than the permeability $\hat{\mu}_{\uparrow}^{*} 100 \%$ from the measurement (in Fig. 6a), no positive permeability of the reversible component can be found to fullfill the criteria defined in the equation (17). In this way, the lower boundary of the ascending curve of the model deviates from the measurement, which make the equivalent permeability of the minor loops (with amplitudes slightly lower than the limiting loop) to be less accurate, as has been highlighted in Fig. 14b on the hysteresis loop with field strength amplitude $\hat{H}_{60 \%}=60 \mathrm{~A} / \mathrm{m}$. This permeability discrepancy together with the coupling from the circuit (e.g. voltage drop on the circuit resistance), adds up to the error of per-cycle energy loss.

Further on, the proposed model is verified on the $3 \mathrm{C} 81$ material from Ferroxcube, the core sample of shape code "TX51/32/19" is taken, while the turns number of both primary and secondary windings are 10 . The parameters are identified based on the limiting hysteresis loop with amplitude $\hat{H}_{100 \%}=100 \mathrm{~A} / \mathrm{m}$ and a symmetrical minor loop with amplitude $\hat{H}_{20 \%}=20 \mathrm{~A} / \mathrm{m}$. The comparison between simulation and measurement is presented in Fig. 16a and Fig. 16b. Significantly different shape of hysteresis loop than N87 is present here and the proposed model is still able to approximate the measurement well. Again the error of equivalent permeability is negligible, as in the case of N87, thanks to the proposed formulation of the reversible component. The maximum error of per-cycle energy loss is $8.7 \%$ at $\hat{H}_{60 \%}=60 \mathrm{~A} / \mathrm{m}$, while the error of all the other loops are under $8 \%$.
The third material chosen for verification is $3 F 3$ from Ferroxcube and the core sample is "TX36/23/15", the turns number of both primary and secondary windings are 8 . The parameters are also identified based on the limiting hysteresis loop with amplitude $\hat{H}_{100 \%}=100 \mathrm{~A} / \mathrm{m}$ and a symmetrical minor loop with amplitude $\hat{H}_{20 \%}=20 \mathrm{~A} / \mathrm{m}$. Again in Fig. $18 \mathrm{a}$ and Fig. 18b, the comparison between simulation and measurement is presented. Larger shape discrepancy than the previous two materials can be observed in Fig. 18a at high field strength amplitudes (e.g. $100 \mathrm{~A} / \mathrm{m}$ ), due to the larger curvature of the 3F3 material's hysteresis loop, while the accuracy of equivalent permeability is still well controlled thanks to the

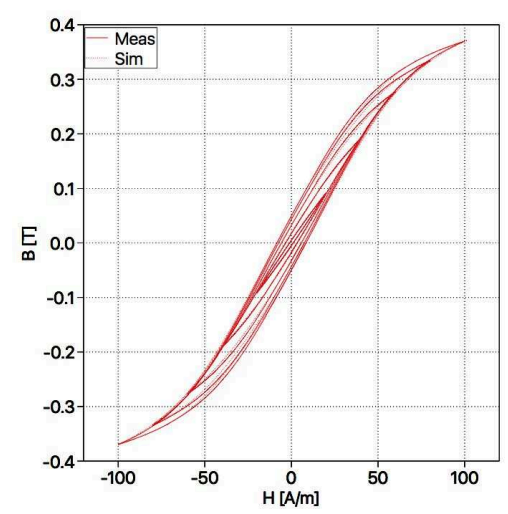

(a)
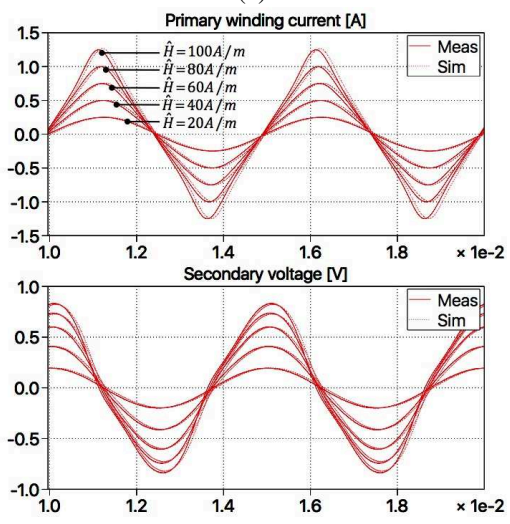

(b)

Fig. 16: Comparison between measurement and simulation of Ferrite 3C81 at different field strength amplitudes (a) Hysteresis loop; (b) Time-domain primary winding current and secondary voltage.

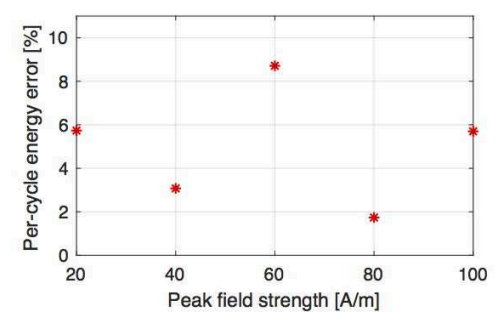

Fig. 17: Percentage error of the simulated per-cycle energy loss from simulation of ferrite $3 \mathrm{C} 81$. 


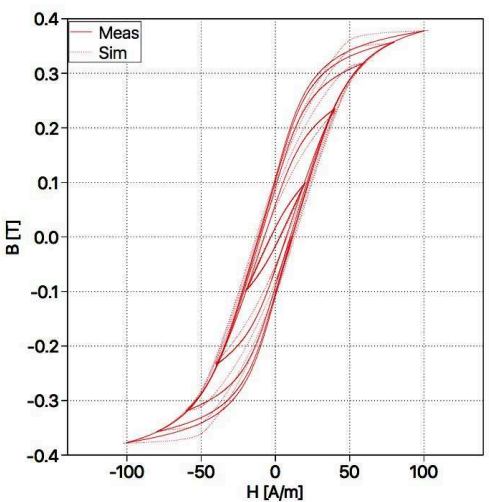

(a)
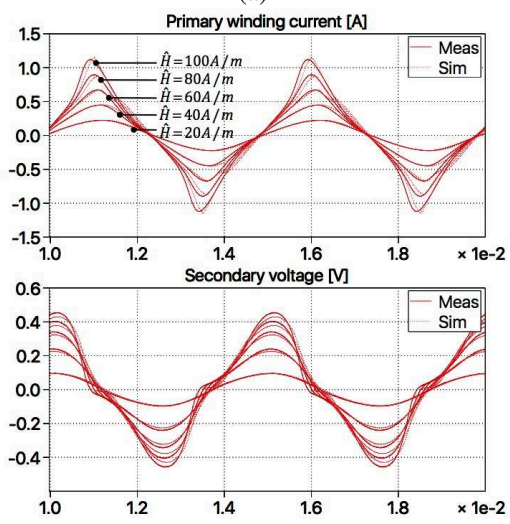

(b)

Fig. 18: Comparison between measurement and simulation of Ferrite 3F3 at different field strength amplitudes (a) Hysteresis loop; (b) Time-domain primary winding current and secondary voltage.

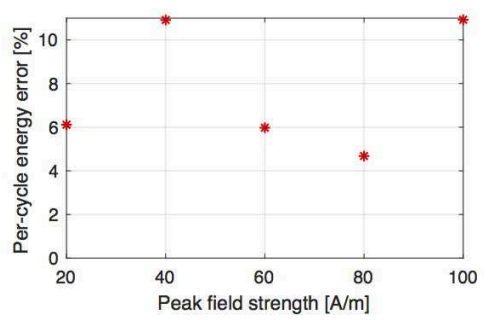

Fig. 19: Percentage error of the simulated per-cycle energy loss from simulation of ferrite $3 \mathrm{~F} 3$.

good approximation of the limiting loops's lower boundary. The error of per-cycle energy loss is illustrated in Fig. 19, the maximum value $10.9 \%$ occurs on the limiting loop with field amplitude $\hat{H}_{100 \%}=100 \mathrm{~A} / \mathrm{m}$.

\section{APPLICATION IN POWER ELECTRONIC CIRCUIT}

In this section, the application of the proposed model in power electronic circuit is demonstrated. Another test setup has been constructed as shown in Fig. 20a and Fig. 20b. The same core sample of $\mathrm{N} 87$ ferrite from the last section is adopted, which is equipped with two windings of eight turns each. On the left-hand side of the primary winding the structure remains the same as the setup introduced in the last section, which is consisted of a power amplifier and current measurement via shunt resistor. On the right-hand side a MOSFET half bridge is additionally connected, whose DC side is supplied by other two power amplifiers. The circuit simulation model is established according to the test setup, as demonstrated on the top of Fig. 20b, including the AC voltage source representing the output of the left-hand side power amplifier, the circuit resistance of $0.4 \Omega$ (measured under DC condition) as well as the MOSFET bridge supplied by two DC sources.

The first scheme tends to imitate the ferrite material's operation in a isolation transformer of a DC-DC converter. The left-hand side power amplifier is muted (output $0 \mathrm{~V}$ voltage), while the MOSFET bridge is operated at $5 \mathrm{kHz}$ switching frequency and $50 \%$ duty cycle, suppose to generate square wave excitation voltage. In this operation condition, the frequency dependent residual effect is negligible. The DC side voltage is

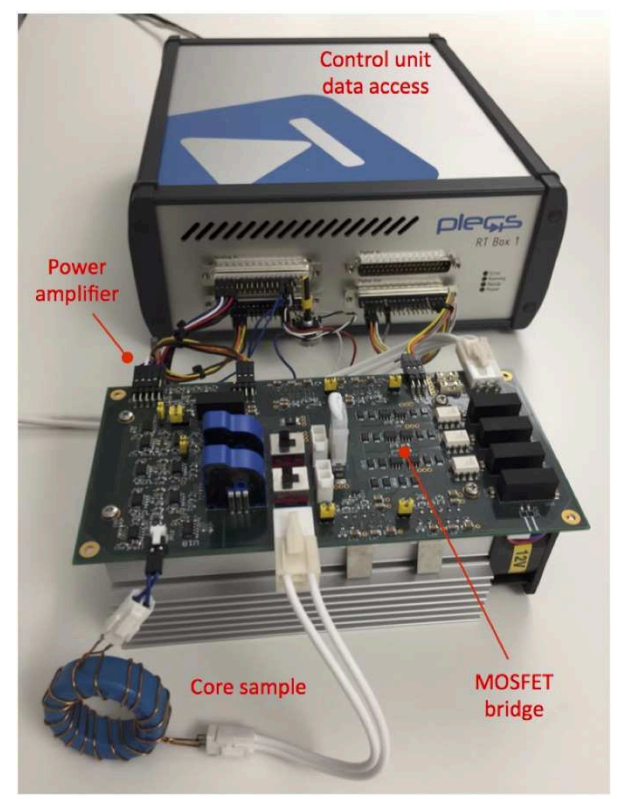

(a)
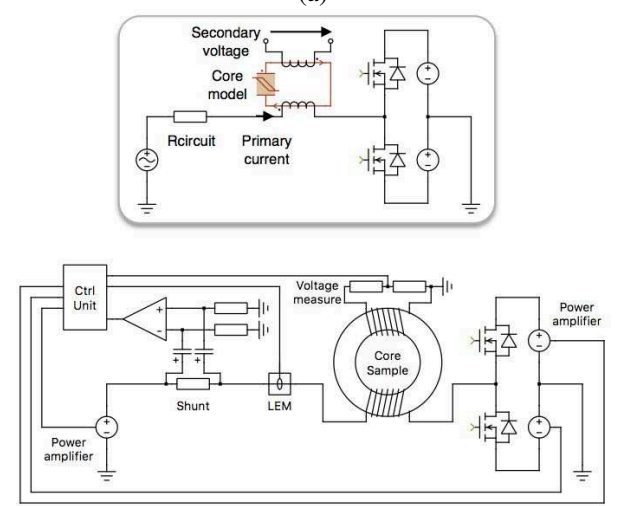

(b)

Fig. 20: (a) Hardware configuration of the test setup with power electronic circuit; (b) Schematic and simulation model of the test setup. 


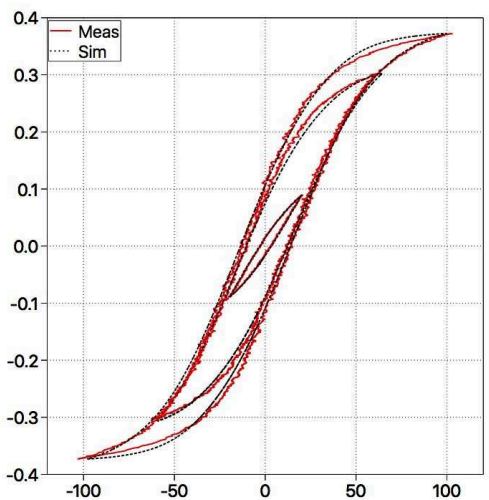

(a)
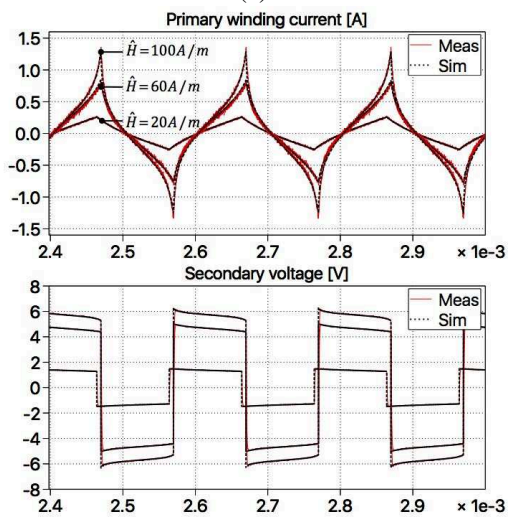

(b)

Fig. 21: Comparison between measurement and simulation of Ferrite N87 in power electronic circuit under 50\% PWM modulation (a) Hysteresis loop; (b) Time-domain primary winding current and secondary voltage.

configured to assume different values, which set the peak field strength of the material at $100 \mathrm{~A} / \mathrm{m}, 60 \mathrm{~A} / \mathrm{m}$ and $20 \mathrm{~A} / \mathrm{m}$, respectively. The simulated B-H characteristic and time domain primary current as well as secondary voltage waveform are compared to the measurement. Due to the material nonlinearity together with the coupling of the circuit resistance, the primary current has obvious harmonic component rather than a ideal triangular wave while the secondary voltage is not ideal square wave, all these effects have been well captured by the proposed model. Since the frequency dependent effect is negligible in this case so that the per-cycle energy loss is mainly determined by the peak field strength, and the error of the per cycle energy loss remains approximately the same value as the corresponding cases verified in the last section (Fig. 12).

The second scheme imitates the condition where the ferrite material is applied for inductor filter operating in a voltage source inverter. The left-hand side power amplifier generates $50 \mathrm{~Hz}$ sinusoidal voltage and the right-hand side MOSFET bridge generates PWM voltage. Sinusoidal voltage with $0.24 \mathrm{~V}$ amplitude and $50 \mathrm{~Hz}$ frequency is generated by the left-hand side power amplifier. The MOSFET bridge is operated under medium switching frequency $2 k H z$ with $50 \%$ duty cycle and DC voltage of $0.24 \mathrm{~V}$.

The simulated hysteresis loop on the B-H plane as well as

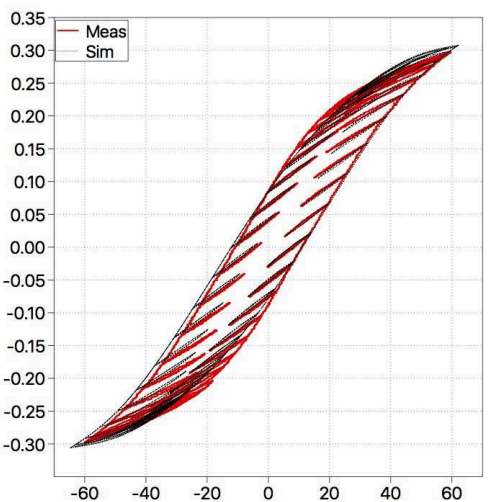

(a)
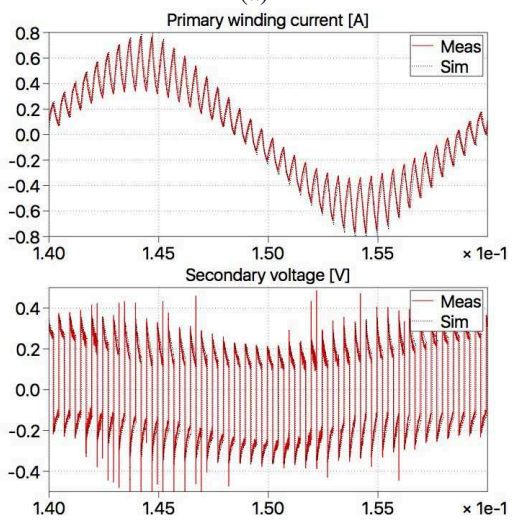

(b)

Fig. 22: Comparison between measurement and simulation of Ferrite N87 in power electronic circuit under sinusoidal and 50\% PWM modulation (a) Hysteresis loop; (b) Time-domain primary winding current and secondary voltage.

the time-domain primary current and secondary open circuit voltage are compared in Fig. 22a and Fig. 22b, respectively. The proposed model is able to reproduce the ripple current and pulsed voltage with good accuracy, where the influence of the circuit resistance has been reflected in a fully coupled way. The sinusoidal voltage of the left-hand side power amplifier contributes to the large hysteresis loop, while the right-hand side MOSFET bridge to the small minor loops. It is to be noted that the minor loops close themselves, which corresponds to the deletion property of the Preisach model as has been discussed in section II. Both loops have been well captured by the proposed model and the error of energy loss per $50 \mathrm{~Hz}$ cycle (including that from both large major- and small minor loops) turns out to be $5 \%$. Please note that there are not only symmetrical- but also asymmetrical hysteresis loops present in this verification scheme. The asymmetrical hysteresis loops are not explicitly controlled during the model parameter identification, nevertheless they are generated by the simulation model as acceptable approximation to the measurement, thanks to the physic-based intrinsic property of the Preisach model.

Finally it is again to be emphasised that the proposed model only covers frequency-independent hysteresis effect of ferrite materials. If the model is parametrised based on the measure- 
ment data obtained at low frequency sinusoidal excitation (e.g. $200 \mathrm{~Hz}$ in this work), the model should be able to predict the core loss in power electronic converters with limited switching frequency, where the static hysteresis effect dominates. According to the experimental measurements conducted, the per-cycle energy loss of several popular ferrite materials under excitation up to $10 \mathrm{kHz}$ (both sinusoidal and PWM) deviates less than $10 \%$ from that obtained under $200 \mathrm{~Hz}$. In this way, the model can be directly applied to high-power applications like medium-voltage grid connected inverters and solid state transformers, where the switching frequency is usually lower than $10 \mathrm{kHz}$. For applications with switching frequency above $10 \mathrm{kHz}$, especially in the case of strongly asymmetrical PWM or with zero-voltage phase (e.g. dual active bridge converter), the frequency dependent part of core loss becomes observable, including the impact of the duty-cycle analysed by [29] as well as the relaxation effect discussed in the work of [30], which arise from residual mechanisms other than static hysteresis. The frequency dependent effects require additional resistive components to be included into the magnetic circuit, which is however not in scope of this work and will be investigated in the future.

\section{ACKNOWLEDGMENT}

This project has been supported in the frame of the ECPE Joint Research Programme.

\section{REFERENCES}

[1] D. Hamill, "Lumped equivalent circuits of magnetic components: the gyrator-capacitor approach," in IEEE Transactions on Power Electronics, vol. 8, 1994, pp. 97-103.

[2] L. Yan and B. Lehman, "A capacitor modeling method for integrated magnetic components in dc/dc converters," in IEEE Transactions on Power Electronics, vol. 20, no. 5, 2005, pp. 987-995.

[3] J. Allmeling, W. Hammer, and J. Schönberger, "Transient simulation of magnetic circuits using the permeance-capacitance analogy," in Control and Modeling for Power Electronics (COMPEL), IEEE 13th Workshop on, 2012.

[4] M. Luo and D. Dujic, "Permeance based modelling of the core corners considering magnetic material nonlinearity," in Annual Conference of the IEEE Industrial Electronics Society (IECON), 2015, pp. 950-955.

[5] M. Luo, D. Dujic, and J. Allmeling, "Leakage flux modeling of multiwinding transformers for system-level simulations," in IEEE Transactions on Power Electronics, vol. 33, no. 3, 2018, pp. 2471-2483.

[6] I. Mayergoyz, Mathematical Models of Hysteresis and Their Applications. Academic Press, 2003.

[7] F. Liorzou, B. Phelps, and D. L. Atherton, "Macroscopic models of magnetization," in IEEE Transactions on Magnetics, 418-428, Ed., vol. 36, no. 2, 2000 .

[8] E. D. Torre, Magnetic Hysteresis. IEEE Press, 1999.

[9] G. Bertotti, F. Fiorillo, and G. P. Soardo, "Dependence of power losses on peak magnetization and magnetization frequency in grain-oriented and non-oriented 3\% sife," in IEEE Transactions on Magnetics, vol. 23, no. 5,1985 , pp. $3520-3522$.

[10] L.-L. Rouve, T. Waeckerle, and A. Kedous-Lebouc, "Application of preisach model to grain oriented steels comparison of different characterizations for the preisach function," in IEEE Transactions on Magnetics, vol. 31, no. 6, 1995, pp. 3557-3559.

[11] B. Azzerboni, E. Cardelli, G. Finocchio, and F. L. Foresta, "Remarks about preisach function approximation using lorentzian function and its identification for non-oriented steels," in IEEE Transactions on Magnetics, vol. 39, no. 5, 2003, pp. 3028-3030.

[12] M. Luo, D. Dujic, and J. Allmeling, "Modelling hysteresis of soft core materials using permeance-capacitance analogy for transient circuit simulations," in European Conference on Power Electronics and Applications 2017 (EPE, ECCE Europe), 2017.
[13] G. Consolo, G. Finocchio, M. Carpentieri, and B. Azzerboni, "A genetic approach to solve numerical problems in the preisach model identification," in IEEE Transactions on Magnetics, vol. 42, no. 5, 2006, pp. $1526-1537$.

[14] E. Cardelli, L. Fiorucci, and E. D. Torre, "Identification of the preisach probability functions for soft magnetic materials," in IEEE Transactions on Magnetics, vol. 37, no. 5, 2001, pp. 3366-3369.

[15] E. Cardelli, G. Finocchio, and E. Pinzaglia, "Increasing the accuracy of the numerical identification of the modified scalar preisach model," in IEEE Transactions on Magnetics, vol. 40, no. 2, 2004, pp. 892-895.

[16] G. Consolo, G. Finocchio, M. Carpentieri, E. Cardelli, and B. Azzerboni, "About identification of scalar preisach functions of soft magnetic materials," in IEEE Transactions on Magnetics, vol. 42, no. 4, 2006, pp. 923-926.

[17] D. H. Everett, "A general approach to hysteresis, part 4-an alternative formulation of the domain model," in Transactions of the Faraday Society, vol. 51, 1955, pp. 1551-1557.

[18] R. M. D. Vecchio, "An efficient procedure for modeling complex hysteresis processes in ferromagnetic materials," in IEEE Transactions on Magnetics, vol. 16, no. 5, 1980, pp. 809-811.

[19] S. R. Naidu, "Simulation of the hysteresis phenomenon using preisach's theory," in IEE Proceedings, vol. 137, no. 2, 1990, pp. 73-79.

[20] J. G. Zhu, S. Y. R. Hui, and V. S. Ramsden, "A dynamic equivalent circuit model for solid magnetic cores for high switching frequency operations," in IEEE Transactions on Power Electronics, vol. 10, no. 6, 1995, pp. 791-795.

[21] S. Y. R. Hui, J. G. Zhu, and V. S. Ramsden, "A generalized dynamic circuit model of magnetic cores for low- and high- frequency applications - part ii: Circuit model formulation and implementation," in IEEE Transactions on Power Electronics, vol. 11, no. 2, 1996, pp. 251-259.

[22] J. T. Hsu and K. D. T. Ngo, "A hammerstein-based dynamic model for hysteresis phenomenon," in IEEE Transactions on Power Electronics, vol. 12 , no. 3, 1997, pp. 406-413.

[23] E. D. Torre, J. Oti, and G. Kadar, "Preisach modeling and reversible magnetization," in IEEE Transactions on Magnetics, vol. 26, no. 6, 1990, pp. 3052-3058.

[24] C. Perez-Rojas, "Fitting saturation and hysteresis via arctangent funcitons," in IEEE Power Engineering Review, vol. 20, no. 1, 2000, pp. $55-57$.

[25] D. C. Jiles and D. L. Atherton, "Ferromagnetic hysteresis," in IEEE Transactions on Magnetics, vol. 19, no. 6, 1983, pp. 2183-2185.

[26] D. C. Jiles, J. B. Thoelke, and M. K. Devine, "Numerial determination of hystereiss parameters for the modeling of magnetic properties using the theory of ferrormangnetic hysteresis," in IEEE Transactions on Magnetics, vol. 28, no. 1, 1992, pp. 27-35.

[27] E. Cardelli, E. D. Torre, and E. Pinzaglia, "Identifying the preisach function for soft magnetic materials," in IEEE Transactions on Magnetics, vol. 39 , no. 3,2003 , pp. 1341-1344.

[28] D. Tan, J. L. Vollin, and S. M. Cuk, "A practical approach for magnetic core-loss characterization," in IEEE Transactions on Power Electronics, vol. 10 , no. 2, 1995, pp. 124-130.

[29] S. Barg, K. Ammous, H. Mejbri, and A. Ammous, "An improved empirical formulation for magnetic core losses estimation under nonsinusoidal induction," in IEEE Transactions on Power Electronics, vol. 32, no. 3, 2017, pp. 2146-2154.

[30] J. Muehlethaler, J. Biela, J. W. Kolar, and A. E. Ecklebe, "Improved core-loss calculation for magnetic components employed in power electronic systems," in IEEE Transactions on Power Electronics, vol. 27, no. 2, 2012, pp. 964-973.

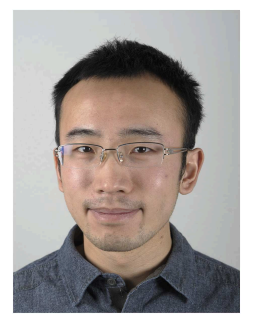

Min Luo Min Luo (M'13) was born in Beijing, China, in 1986. He received the B.S. degree in electrical engineering from Tsinghua University, Beijing, China, in 2009 and M.S. degree in electrical power engineering from RWTH Aachen University, Aachen, Germany, in 2012. Since 2012 he has been with Plexim as application engineer, working on the software PLECS for fast simulation of power electronic systems. In 2014 he joined Power Electronics Laboratory of École Polytechnique Fédérale de Lausanne (EPFL) in Lausanne, Switzerland as external doctoral research assistant, pursuing his $\mathrm{PhD}$ degree. His current research interest include dynamic modelling of magnetic components and real-time HIL simulation of power converters. 


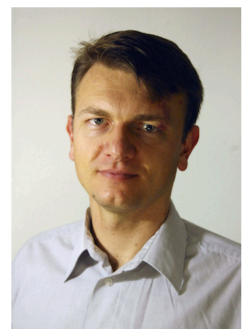

Drazen Dujic Drazen Dujic (S'03-M'09-SM'12) received the Dipl.-Ing. and M.Sc. degrees from the University of Novi Sad, Novi Sad, Serbia, in 2002 and 2005, respectively, and the Ph.D. degree from the Liverpool John Moores University, Liverpool, U.K., in 2008, all in electrical engineering. From 2002 to 2006, he was with the Department of Electrical Engineering, University of Novi Sad as a Research Assistant, and from 2006 to 2009 with Liverpool John Moores University as a Research Associate. From 2009 till 2013, he was with ABB Corporate Research Centre, Switzerland, as a Principal Scientist working on the power electronics projects spanning the range from low-voltage/power SMPS in below kilowatt range to medium voltage high-power converters in a megawatt range. During 2010-2011, he was a member of a project team responsible for the development of the worlds first power electronic traction transformer (PETT) successfully commissioned on the locomotive. From 2013 till 2014, he was with ABB Medium Voltage Drives, Turgi, Switzerland, as R\&D Platform Manager, responsible for ABB's largest IGCT based medium voltage drive - ACS6000. He is currently with Ecole Polytechnique Federale de Lausanne EPFL, Lausanne, Switzerland, as an Assistant Professor and the Director of the Power Electronics Laboratory. His current research interests include the areas of design and control of advanced high-power electronics systems and high performance drives. He has authored or coauthored more than 80 scientific publications and has filed eleven patents. He is an Associate Editor for IEEE Transactions on Industrial Electronics, IEEE Transaction on Power Electronics and IET Electric Power Applications. He has received the First Prize Paper Award by the Electric Machines Committee of the IEEE Industrial Electronics Society at IECON-2007. In 2014 he has received the Isao Takahashi Power Electronics Award for outstanding achievement in power electronics.

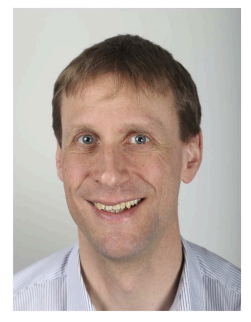

Jost Allmeling Jost Allmeling ( $\mathrm{S}^{\prime} 98-\mathrm{M}^{\prime} 03$ ) was born in Hamburg, Germany, in 1972. He received the M.S. degree in electrical engineering from Aachen University, Aachen, Germany, in 1996 and the Ph.D. degree from the Swiss Federal Institute of Technology (ETH), Zurich, Switzerland, in 2001. In 1996, he became a Research Associate at the Power Electronics Laboratory at ETH. From 2001 until 2003, he was with the Power Systems Laboratory at ETH, as a Post-Doctoral Researcher. In 2002 , Jost co-founded Plexim, a spin-off company from ETH Zurich that develops the software PLECS for fast simulation of power electronic systems. Currently, he is the Managing Director of Plexim. His research interests include simulation of power electronics, modeling electrical and mechanical components, real-time HIL simulation and inverter control. 